\title{
A NEW UPPER BOUND FOR THE LAPLACIAN SPECTRAL RADIUS OF A GRAPH*
}

\author{
AIMEI YU ${ }^{\dagger}$
}

Abstract. Let $G$ be a simple connected graph with $m$ edges, and the line graph of $G$ with degree sequence $t_{1} \geq t_{2} \geq \cdots \geq t_{n}$. This paper presents a new upper bound for the Laplacian spectral radius of $G$ as follows:

$$
\mu_{1}(G) \leq \min _{1 \leq i \leq m}\left\{\frac{t_{i}+3+\sqrt{\left(t_{i}+1\right)^{2}+4(i-1)\left(t_{1}-t_{i}\right)}}{2}\right\} .
$$

Key words. Laplacian spectral radius, Line graph, Degree sequence.

AMS subject classifications. 05C50, 15A18.

1. Introduction. Let $G=(V(G), E(G))$ be a simple undirected graph with $n$ vertices, $m$ edges and degree sequence $d_{1}, d_{2}, \ldots, d_{n}$, where $d_{i}=d\left(v_{i}\right)$, the degree of $v_{i}$, is the number of edges incident with $v_{i}$, for $v_{i} \in V(G)$. Let $A(G)$ be the adjacency matrix of $G$ and $D(G)$ be the diagonal matrix of vertex degrees. Then the Laplacian matrix of $G$ is $L(G)=D(G)-A(G)$.

Let $G^{*}$ be the line graph of $G$, and denote by $B(G)$ the adjacency matrix of $G^{*}$. It is easy to see that $B(G)$ is a real symmetric matrix, so its eigenvalues are real numbers. Note that the edges of $G$ are the vertices of $G^{*}$. If $e_{l}=v_{i} v_{j} \in E(G)$, then the degree of $e_{l}$ in $G^{*}$ is $d_{i}+d_{j}-2$, i.e., the row sum of $B(G)$ corresponding to $e_{l}$. Denote the $i$ th row sum of $B(G)$ by $t_{i}(1 \leq i \leq m)$. Without loss of generality, we assume $t_{1} \geq t_{2} \geq \cdots \geq t_{m}$.

Note that $L(G)$ is positive semidefinite, symmetric and singular. Without loss of generality, we assume that $\mu_{1}(G) \geq \mu_{2}(G) \geq \cdots \geq \mu_{n-1}(G) \geq \mu_{n}(G)=0$ are the eigenvalues of $L(G)$. The eigenvalues of the $L(G)$, especially the largest and the second smallest eigenvalues, are important in graph theory, because they have relations to numerous graph invariants including the connectivity, expandability, isoperimetric number, maximum cut, genus, diameter and bandwidth-type parameters of a graph (see, for example, $[1,4,7]$ and the references therein).

\footnotetext{
${ }^{*}$ Received by the editors on April 25, 2010. Accepted for publication on November 15, 2010. Handling Editor: Bryan Shader.

${ }^{\dagger}$ Department of Mathematics, Beijing Jiaotong University, Beijing 100044, China (yuaimeimath@yeah.net). Supported by Specialized Research Fund for the Doctoral Program of Higher Education of China (No. 200800041001).
} 
The largest eigenvalue of $L(G)$ is called the Laplacian spectral radius of $G$. In many applications, one needs good bounds for the Laplacian spectral radius $\mu_{1}(G)$. The following are some known upper bounds for $\mu_{1}(G)$.

1. (Anderson and Morley [2]):

$$
\mu_{1}(G) \leq 2+t_{1}
$$

where equality holds if and only if $G$ is a regular or semiregular bipartite graph.

2. (Li and Zhang $[6,9])$ :

$$
\mu_{1}(G) \leq 2+\sqrt{t_{1} t_{2}}
$$

where equality holds if and only if $G$ is a regular bipartite graph or semiregular bipartite graph or the path with four vertices.

3. (Das $[5,12])$ :

$$
\mu_{1}(G) \leq \max \left\{\left|N\left(v_{i}\right) \cup N\left(v_{j}\right)\right|: 1 \leq i<j \leq n, v_{i} v_{j} \in E(G)\right\},
$$

and equality holds if and only if $G \cong F^{+}$, where $F^{+}$is the supergraph of a semiregular graph $F=(U, W ; E(F))$ with the following property: if $u v \in E\left(F^{+}\right)$, then either $u v \in E(F)$ or $u, v \in U$ (resp. $W$ ) with $N_{W}(u)=N_{W}(v)\left(\operatorname{resp} . N_{U}(u)=N_{U}(v)\right)$.

It is easy to see that the upper bounds (1.2) and (1.3) are both the improvements of bound (1.1). But bounds in (1.2) and (1.3) are incomparable (see [10]). In the paper, we give a new upper bound of the Laplacian spectral radius of $G$ as follows:

$$
\mu_{1}(G) \leq \min _{1 \leq i \leq m}\left\{\frac{t_{i}+3+\sqrt{\left(t_{i}+1\right)^{2}+4(i-1)\left(t_{1}-t_{i}\right)}}{2}\right\},
$$

which is better than the bound (1.2) in many cases, and is incomparable with the bound (1.3).

2. Lemmas and results. We first give some lemmas that will be used in our proof.

For a matrix $M$, we denote its largest eigenvalue by $\lambda_{1}(M)$. It is easy to see that for a graph $G, \mu_{1}(G)=\lambda_{1}(L(G))$.

LEMma 2.1. [8] Let $A$ be a nonnegative irreducible $n \times n$ matrix with largest eigenvalue $\lambda_{1}(A)$ and row sums $s_{1}, s_{2}, \ldots, s_{n}$. Then

$$
\min _{1 \leq i \leq n} s_{i} \leq \lambda_{1}(A) \leq \max _{1 \leq i \leq n} s_{i}
$$

Moreover, each of the equalities holds if and only if the row sums of $A$ are all equal. 
Lemma 2.2. [13] Let $G$ be a connected graph. Then

$$
\mu_{1}(G) \leq 2+\lambda_{1}(B(G))
$$

and equality holds if and only if $G$ is a bipartite graph.

From Lemma 2.1 and Lemma 2.2, we can obtain the following upper bound of $\mu_{1}(G)$.

Lemma 2.3. [2] Let $G$ be a connected graph. Then

$$
\mu_{1}(G) \leq 2+t_{1}
$$

Let $G=(V(G), E(G))$ be a graph. A subset $S$ of $V(G)$ is called an independent set of $G$ if there are no two vertices of $S$ are adjacent in $G$. An independent set is maximum if $G$ has no independent set $S^{\prime}$ with $\left|S^{\prime}\right|>|S|$. The number of vertices in a maximum independent set of $G$ is called the independence number of $G$ and is denoted by $\alpha(G)$. A subset $K$ of $V(G)$ is a called a covering of $G$ if every edge of $G$ has at least one end in $K$. A covering $K$ is a minimum covering if $G$ has no covering $K^{\prime}$ with $\left|K^{\prime}\right|<|K|$. The number of vertices in a minimum covering of $G$ is the covering number of $G$ and is denoted by $\beta(G)$.

LEMma 2.4. [3] Let $G$ be a graph with $n$ vertices. Then

$$
\alpha(G)+\beta(G)=n .
$$

Lemma 2.5. Let $P_{2}^{k, k}(k \geq 1)$ be the graph as shown in Fig. 1. Then

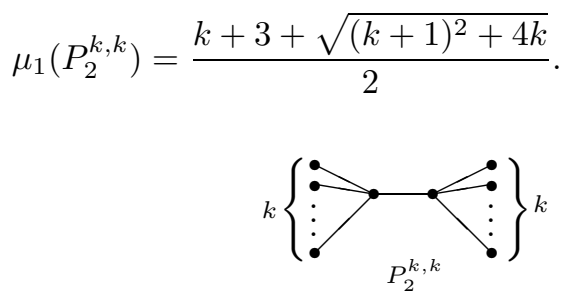

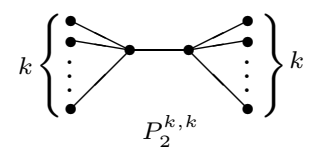

Fig. 1

Proof. From equality (S6) in [11], we have the characteristic polynomial of $L\left(P_{2}^{k, k}\right)$ is

$$
x(x-1)^{2 k-2}\left[x^{3}-(2 k+4) x^{2}+\left(k^{2}+4 k+5\right) x-2 k-2\right] .
$$

Solving the equation $x^{3}-(2 k+4) x^{2}+\left(k^{2}+4 k+5\right) x-2 k-2=0$, yields

$$
x=1+k \text { or } x=\frac{k+3+\sqrt{(k+1)^{2}+4 k}}{2} \text { or } \frac{k+3-\sqrt{(k+1)^{2}+4 k}}{2} .
$$


Note that $\mu_{1}\left(P_{2}^{k, k}\right)$ is the largest root of the characteristic polynomial of $L\left(P_{2}^{k, k}\right)$ and

$$
\frac{k+3+\sqrt{(k+1)^{2}+4 k}}{2} \geq k+2 .
$$

It is easy to see that

$$
\mu_{1}\left(P_{2}^{k, k}\right)=\frac{k+3+\sqrt{(k+1)^{2}+4 k}}{2}
$$

Now we begin to show our main results.

THEOREM 2.6. Let $G$ be a connected graph. Then

$$
\mu_{1}(G) \leq \frac{t_{i}+3+\sqrt{\left(t_{i}+1\right)^{2}+4(i-1)\left(t_{1}-t_{i}\right)}}{2}
$$

where $1 \leq i \leq m$. When $i=1$, the equality holds if and only if $G$ is a regular or semiregular bipartite graph. When $2 \leq i \leq m$, the equality holds if and only if $i=2$ and $G \cong P_{2}^{k, k}$ (as shown in Fig. 1).

Proof. If $i=1$ or $t_{i}=t_{1}$, then the inequality (2.1) is

$$
\mu_{1}(G) \leq 2+t_{1}
$$

By Lemma 2.3, it is true and the equality holds if and only if $G$ is a regular or semiregular bipartite graph.

Now suppose that $2 \leq i \leq m$ and $t_{1} \geq \cdots \geq t_{i-1}>t_{i} \geq \cdots \geq t_{m}$.

Let $B=\left(b_{i j}\right)_{m \times m}$ be the adjacency matrix of the line graph of $G$. We can write $B$ as

$$
\left(\begin{array}{ll}
B_{11} & B_{12} \\
B_{21} & B_{22}
\end{array}\right)
$$

where $B_{11}$ is an $(i-1) \times(i-1)$ matrix and $B_{22}$ is a $(m-i+1) \times(m-i+1)$ matrix. Let

$$
U=\left(\begin{array}{cc}
x I_{i-1} & 0 \\
0 & I_{m-i+1}
\end{array}\right),
$$

where $x>1, I_{i-1}$ is an $(i-1) \times(i-1)$ unit matrix and $I_{m-i+1}$ is a $(m-i+1) \times(m-i+1)$ unit matrix. Then

$$
B^{\prime}=U^{-1} B U=\left(\begin{array}{cc}
B_{11} & \frac{1}{x} B_{12} \\
x B_{21} & B_{22}
\end{array}\right)
$$


and $B$ are similar matrices and so they have the same eigenvalues. In particular, $\lambda_{1}(B)=\lambda_{1}\left(B^{\prime}\right)$. Let $s_{j}\left(B^{\prime}\right)(1 \leq j \leq m)$ be the row sum of $B^{\prime}$, respectively. Then we have the following results.

For $1 \leq l \leq i-1$,

$$
\begin{aligned}
s_{l}\left(B^{\prime}\right) & =\sum_{j=1}^{i-1} b_{l j}+\frac{1}{x} \sum_{j=i}^{m} b_{l j}=\frac{1}{x} \sum_{j=1}^{m} b_{l j}+\left(1-\frac{1}{x}\right) \sum_{j=1}^{i-1} b_{l j} \\
& =\frac{1}{x} t_{l}+\left(1-\frac{1}{x}\right) \sum_{j=1}^{i-1} b_{l j} .
\end{aligned}
$$

For $i \leq k \leq m$,

$$
\begin{aligned}
s_{k}\left(B^{\prime}\right) & =x \sum_{j=1}^{i-1} b_{k j}+\sum_{j=i}^{m} b_{k j}=\sum_{j=1}^{m} b_{k j}+(x-1) \sum_{j=1}^{i-1} b_{k j} \\
& =t_{k}+(x-1) \sum_{j=1}^{i-1} b_{k j} .
\end{aligned}
$$

Since $x>1$ and $t_{1} \geq \cdots \geq t_{i-1}>t_{i} \geq \cdots \geq t_{m}$, we have

$$
s_{l}\left(B^{\prime}\right) \leq \frac{1}{x} t_{1}+\left(1-\frac{1}{x}\right)(i-2) \quad(1 \leq l \leq i-1)
$$

and

$$
s_{k}\left(B^{\prime}\right) \leq t_{i}+(x-1)(i-1) \quad(i \leq k \leq m)
$$

It is easy to see that

$$
\max _{1 \leq j \leq m} s_{j}\left(B^{\prime}\right) \leq \max \left\{\frac{1}{x} t_{1}+\left(1-\frac{1}{x}\right)(i-2), t_{i}+(x-1)(i-1)\right\} .
$$

The solution to

$$
\frac{1}{x} t_{1}+\left(1-\frac{1}{x}\right)(i-2)=t_{i}+(x-1)(i-1) .
$$

is

$$
\begin{aligned}
x & =\frac{2 i-3-t_{i}+\sqrt{\left(2 i-3-t_{i}\right)^{2}+4(i-1)\left(t_{1}-i+2\right)}}{2(i-1)} \\
& =\frac{2 i-3-t_{i}+\sqrt{\left(t_{i}+1\right)^{2}+4(i-1)\left(t_{1}-t_{i}\right)}}{2(i-1)} .
\end{aligned}
$$


Since $i \geq 2$ and $t_{1}>t_{i}$, we have $x>1$. Hence, by Lemma 2.2, we have

$$
\begin{aligned}
\mu_{1}(G) & \leq 2+\lambda_{1}(B)=2+\lambda_{1}\left(B^{\prime}\right) \\
& \leq 2+t_{i}+(x-1)(i-1) \\
& =2+\frac{t_{i}-1+\sqrt{\left(t_{i}+1\right)^{2}+4(i-1)\left(t_{1}-t_{i}\right)}}{2} \\
& =\frac{t_{i}+3+\sqrt{\left(t_{i}+1\right)^{2}+4(i-1)\left(t_{1}-t_{i}\right)}}{2} .
\end{aligned}
$$

In order for the equality to hold, each of the above inequalities must be equality. From Lemma 2.2, $G$ is a bipartite graph. From (2.2), we have that $t_{l}=t_{1}$ when $1 \leq l \leq i-1$ and $b_{l j}=1$ when $1 \leq l \leq i-1,1 \leq j \leq i-1, l \neq j$. From (2.3), we have that $t_{k}=t_{i}$ and $b_{k j}=1$ when $i \leq k \leq m$ and $1 \leq j \leq i-1$. Thus $b_{k j}=1$ when $1 \leq k \leq m, 1 \leq j \leq i-1$ and $k \neq j$, which implies $t_{1}=m-1$. So $m-1=t_{1}=\cdots=t_{i-1}>t_{i}=t_{i+1}=\cdots=t_{m}$. Since $t_{1}=m-1$, there must be an edge $e$ of $G$ such that each edge of $G$ is incident with the ends of $e$. So $\beta(G) \leq 2$. From Lemma 2.4, we have $\alpha(G) \geq n-2$. Noting that $G$ is a bipartite graph and $m-1=t_{1}=\cdots=t_{i-1}>t_{i}=t_{i+1}=\cdots=t_{m}$, we have $i=2$ and $G \cong P_{2}^{k, k}$ (as shown in Fig. 1), where $k \geq 1$ and $2 k+2=n$.

Conversely, if $G \cong P_{2}^{k, k}$, then $t_{1}=2 k$ and $t_{i}=k$ for $2 \leq i \leq m$. Take $i=2$, then

$$
\frac{t_{i}+3+\sqrt{\left(t_{i}+1\right)^{2}+4(i-1)\left(t_{1}-t_{i}\right)}}{2}=\frac{k+3+\sqrt{(k+1)^{2}+4 k}}{2}=\mu_{1}(G) .
$$

Denote by $\Delta\left(G^{*}\right)$ and $\Delta^{\prime}\left(G^{*}\right)$ the maximum degree and second largest degree of $G^{*}$, respectively. Let $p$ be the number of vertices of $G^{*}$ with the maximum degree $\Delta\left(G^{*}\right)$. If $G^{*}$ is a regular graph, we let $\Delta\left(G^{*}\right)=\Delta^{\prime}\left(G^{*}\right)$.

Corollary 2.7. Let $G$ be a connected graph. Denote by $G^{*}$ the line graph of $G$, and denote by $\Delta\left(G^{*}\right)$ and $\Delta^{\prime}\left(G^{*}\right)$ the maximum degree and second largest degree of $G^{*}$, respectively. If there are $p$ vertices of $G^{*}$ with the maximum degree $\Delta\left(G^{*}\right)$, then

$$
\mu_{1}(G) \leq \frac{\Delta^{\prime}\left(G^{*}\right)+3+\sqrt{\left(\Delta^{\prime}\left(G^{*}\right)+1\right)^{2}+4 p\left(\Delta\left(G^{*}\right)-\Delta^{\prime}\left(G^{*}\right)\right)}}{2} .
$$

Proof. The result holds from Theorem 2.6 by taking $\Delta\left(G^{*}\right)=t_{1}, \Delta^{\prime}\left(G^{*}\right)=t_{i}$ and $p=i-1$.

REMARK 2.8. From Corollary 2.7, we have

$$
\mu_{1}(G) \leq \frac{\Delta\left(G^{*}\right)+\Delta^{\prime}\left(G^{*}\right)}{2}+2,
$$


when $4 p \leq \Delta\left(G^{*}\right)+\Delta^{\prime}\left(G^{*}\right)+2$.

Based on Theorem 2.6, we obtain a stronger result.

THEOREM 2.9. Let $G$ be a connected graph with $m$ edges. Then

$$
\mu_{1}(G) \leq \min _{1 \leq i \leq m}\left\{\frac{t_{i}+3+\sqrt{\left(t_{i}+1\right)^{2}+4(i-1)\left(t_{1}-t_{i}\right)}}{2}\right\}
$$

REMARK 2.10. The bound (2.4) is incomparable with the bound (1.3). As illustrations, we consider two connected graphs $G_{1}$ and $G_{2}$ as shown in Fig. 2. Note that the degree sequences of the line graph of $G_{1}$ and $G_{2}$ are $(7,6,5,4,4,4,3,3,2)$ and $(4,4,4,4,4,3,3,2)$, respectively.
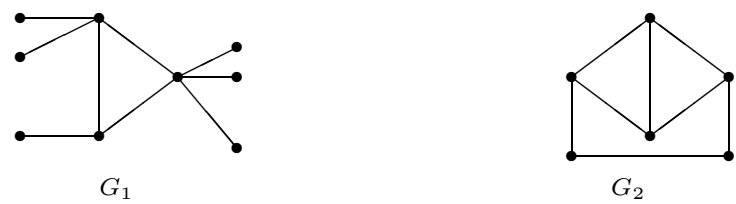

Fig. 2

From inequalities (1.2), (1.3) and (2.4), we get different bounds of $\mu_{1}(G)$ shown in the following table. It is easy to see that if $G \cong G_{1}$, the bound (2.4) is better than the bound (1.3) and if $G \cong G_{2}$, the bound (1.3) is better than the bound (2.4).

\begin{tabular}{|c|c|c|c|}
\hline & $(1.2)$ & $(1.3)$ & $(2.4)$ \\
\hline$G_{1}$ & 8.48 & 8 & 7.41 \\
\hline$G_{2}$ & 6 & 5 & 6 \\
\hline
\end{tabular}

From graphs $G_{1}$ and $G_{2}$, we find that the bound (2.4) is no worse than the bound (1.2). In fact, we can show that for any connected graphs. Moreover, we can show that the bound (2.4) is better than the bound (1.2) in many cases.

If $t_{1}=t_{2}$, from inequality (1.2), we have $\mu_{1}(G) \leq t_{1}+2$. From inequality (2.4), we have

$$
\begin{aligned}
\mu_{1}(G) & \leq \min _{1 \leq i \leq m}\left\{\frac{t_{i}+3+\sqrt{\left(t_{i}+1\right)^{2}+4(i-1)\left(t_{1}-t_{i}\right)}}{2}\right\} \\
& \leq \frac{t_{1}+3+\sqrt{\left(t_{1}+1\right)^{2}+4(1-1)\left(t_{1}-t_{1}\right)}}{2} \\
& =t_{1}+2 .
\end{aligned}
$$


In this case, the bound (2.4) is no worse than the bound (1.2).

If $t_{1}>t_{2}$, from inequality $(1.2)$, we have $\mu_{1}(G) \leq 2+\sqrt{t_{1} t_{2}}$. From inequality (2.4), we have

$$
\begin{aligned}
\mu_{1}(G) & \leq \min _{1 \leq i \leq m}\left\{\frac{t_{i}+3+\sqrt{\left(t_{i}+1\right)^{2}+4(i-1)\left(t_{1}-t_{i}\right)}}{2}\right\} \\
& \leq \frac{t_{2}+3+\sqrt{\left(t_{2}+1\right)^{2}+4\left(t_{1}-t_{2}\right)}}{2} .
\end{aligned}
$$

It is not difficult to show

$$
\frac{t_{2}+3+\sqrt{\left(t_{2}+1\right)^{2}+4\left(t_{1}-t_{2}\right)}}{2} \leq 2+\sqrt{t_{1} t_{2}} .
$$

Assume

$$
\frac{t_{2}+3+\sqrt{\left(t_{2}+1\right)^{2}+4\left(t_{1}-t_{2}\right)}}{2}>2+\sqrt{t_{1} t_{2}} .
$$

Then we have

$$
\sqrt{\left(t_{2}+1\right)^{2}+4\left(t_{1}-t_{2}\right)}>1-t_{2}+2 \sqrt{t_{1} t_{2}} .
$$

Note that

$$
1-t_{2}+2 \sqrt{t_{1} t_{2}}>1-t_{2}+2 \sqrt{t_{2} t_{2}}=1+t_{2}>0,
$$

we have

$$
\left(t_{2}+1\right)^{2}+4\left(t_{1}-t_{2}\right)>\left(1-t_{2}\right)^{2}+4 t_{1} t_{2}+4 \sqrt{t_{1} t_{2}}\left(1-t_{2}\right),
$$

i.e.,

$$
4 \sqrt{t_{1}}\left(\sqrt{t_{1}}-\sqrt{t_{2}}\right)\left(1-t_{2}\right)>0 .
$$

Note that $t_{1}>t_{2} \geq 1$, and we get a contradiction. So in this case, the bound (2.4) is also no worse than the bound (1.2). From above proof, it is not difficult to see that the bound (2.4) is better than the bound (1.2) if $t_{1}>t_{2} \geq 2$.

\section{REFERENCES}

[1] N. Alon. Eigenvalues and expanders. Combinatorica, 6:83-96, 1986.

[2] W.N. Anderson and T.D. Morley. Eigenvalues of the Laplacian of a graph. Linear and Multilinear Algebra, 18:141-145, 1985.

[3] J.A. Bondy and U.S.R. Murty. Graph Theory with Applications. American Elsevier Publishing Co., Inc., New York, 1976. 
[4] F.R.K. Chung. Eigenvalues of graphs. Proceeding of the International Congress of Mathematicians. Birkhäuser, Basel, Switzerland, 1995, pp.1333-1342.

[5] K.Ch. Das. An improved upper bound for Laplacian graph eigenvalues. Linear Algebra and its Applications, 368:269-278, 2003.

[6] J.S. Li and X.D. Zhang. On the Laplacian eigenvalues of a graph. Linear Algebra and its Applications, 285:305-307, 1998.

[7] R. Merris. Laplacian matrices of graphs: a survey. Linear Algebra and its Applications, 197198:143-176, 1994

[8] H. Minc. Nonnegative Matrices. John Wiley \& Sons, Inc., New York, 1988.

[9] Y.L. Pan. Sharp upper bounds for the Laplacian graph eigenvalues. Linear Algebra and its Applications, 355:287-295, 2002.

[10] O. Rojo, R. Soto, and H. Rojo. An always nontrivial upper bound for Laplacian graph eigenvalues. Linear Algebra and its Applications, 312:155-159, 2000.

[11] A.M. Yu, M. Lu, and F. Tian. Ordering trees by their Laplacian radii. Linear Algebra and its Applications, 405:45-59, 2005.

[12] A.M. Yu, M. Lu, and F. Tian. Characterization on graphs which achieve a Das' upper bound for Laplacian spectral radius. Linear Algebra and its Applications, 400:271-277, 2005.

[13] X.D. Zhang and R. Luo. The spectral radius of triangle-free graphs. Australasian Journal of Combinatorics, 26:33-39, 2002. 